\title{
Strukturentwicklungspläne und Zielvereinbarungen
}

\author{
Orientierungen für strategische Planungen
}

\section{Einleitung}

Strategie bildet im Hochschulkontext einen Gegenstand differenzierter Diskussionen. Neben vielen weiteren Aspekten finden Erörterungen zu den Potenzialen von Strategie hinsichtlich der Ausrichtung der Institutionen auf Modernisierung oder Profilierung (Dräger et al. 2017), der Wettbewerbsfähigkeit (Geiger 2011, 44-48), der Entwicklung zukunftsweisender Kompetenzen (Meyer-Guckel et al. 2019) oder der Ausformungen von Profiltypen wie Internationalisierung, anwendungs- oder forschungsorientierter Lehre oder Lebenslangem Lernen (Schmid/Baeßler 2016) statt. In der Fülle der Möglichkeiten der Ausformulierung von Strategie weisen die Dokumentenformen der Entwicklungs- und Strukturpläne (ESP) sowie Zielvereinbarungen von Hochschulen die Besonderheiten auf, in einem institutionalisierten Rahmen zu entstehen, von den zuständigen Hochschulgremien und gegebenenfalls den entsprechenden politischen Instanzen offiziell beschlossen $\mathrm{zu}$ werden sowie für die jeweilige Hochschule in ihrer Gesamtheit von Gültigkeit zu sein. Sie dienen somit intern der strategischen Orientierung, extern der Positionierung in Wissenschaftsbetrieb und Gesellschaft sowie in beide Stoßrichtungen der Demonstration des akademischen Selbstverständnisses. Dabei besteht der Charakter dieser Dokumente in einer weit gefassten, abstrakten, grundsätzliche strategische Linien vorgebenden Klammer, die in der konkreten Ausformung der Hochschulpraxis spätestens mit dem Gebot der Freiheit von Forschung und Lehre (Grundgesetz, Art. 5, Abs. 3, Satz 1) an ihre Grenzen stößt.

Im Zuge des Forschungsprojekts Lernwelt Hochschule soll mit der Untersuchung von Entwicklungs- und Strukturplänen sowie Zielvereinbarungen deutscher Hochschulen der Versuch unternommen werden, einen Abgleich der in den einzelnen Erhebungsschritten des Projekts (Online-Befragung, Leitfadeninterviews, Fallstudien) generierten Ergebnissen mit der sich in jenen Dokumenten manifestierenden offiziellen strategischen Positionierung der Institutionen zu ermöglichen. Dabei wird es nicht darum gehen, diesen Abgleich für jede einzelne Hochschule $\mathrm{zu}$ vollziehen, sondern anhand der Quellenlage generelle 
strategische Strukturen und Trends innerhalb der deutschen Hochschullandschaft aufzuzeigen.

Zur Definition des zugrundeliegenden Primärquellenmaterials soll die Terminologie in Kürze in den Blick genommen werden. Das vielfältige Vokabular von Entwicklungsplan über Strukturplan, Hochschulentwicklungsplan bis hin zu Strukturentwicklungsplan soll dabei unter dem Akronym ESP firmieren. Weder in den konkreten Dokumenten, noch von institutioneller Seite werden aktuelle ESP-Definitionen vorgenommen, so dass an dieser Stelle auf folgende Begriffsfassung rekurriert wird:

Hochschulentwicklungsplan: nach 1990 zunehmend an Hochschulen ausgearbeitetes strategisches Instrument zur Definition der Ziele und Leistungen der eigenen Organisation. Der H. dient als Planungsgrundlage und wird regelmäßig fortgeschrieben, in neueren Hochschulgesetzen z. T. gesetzlich gefordert. (Tenorth/Tippelt 2007, 32)

Ein ähnliches Verständnis drückt sich in der knappen Bestimmung aus, die Hochschulentwicklungsplanung diene „dem Ziel, eine abgestimmte und bedarfsgerechte Entwicklung der Hochschule zu gewährleisten“ (Schmuck 2010, 42). Bertholds Feststellung, eine erschöpfende Untersuchung des Gegenstandes ESP habe im deutschsprachigen Kontext noch nicht stattgefunden (Berthold 2011, 56), besitzt unverändert Gültigkeit. Es kann jedoch festgehalten werden, dass die überwiegende Zahl der deutschen Hochschulen per Landeshochschulgesetzgebung zur Ausfertigung eines ESPs verpflichtet ist (Scherm et al. 2014, 102). Der Einzug dieses im wesentlichen Management- und Steuerungsfunktionen erfüllenden Instruments in die Kontinuität der Hochschulleitungsprozesse scheint dabei seitens der derartigen Eingriffen nicht immer mit gleicher Offenheit gegenüberstehenden Gremien und Entscheidungsträgern im Allgemeinen akzeptiert (Palandt 2015, 46). Grundsätzlich möglich ist die Existenz eines in Ergänzung zu den hochschulspezifischen ESPs geltenden Hochschulentwicklungsplan eines Bundeslandes, im Rahmen des Forschungsprojektes Lernwelt Hochschule erfolgte allerdings eine Konzentration auf die erstgenannten Dokumente, da auch in den übrigen Erhebungsschritten einzelne Einrichtungen und nicht Bundesländer als Ganzes in den Blick genommen wurden.

Parallel dazu erfolgt die Analyse auch ausschließlich für die Zielvereinbarungen, die zwischen einer spezifischen Hochschule und dem Bundesland, in dessen Trägerschaft sich diese Hochschule befindet, geschlossen wurden, während hochschulinterne Zielvereinbarungen mit etwa einzelnen Fakultäten oder Abteilungen sowie landesweite Zielvereinbarungen nicht berücksichtigt werden. Für Zielvereinbarungen identifiziert Berthold fünf Konstitutionsmerkmale: der verbindliche Abschluss durch zwei Parteien, namentlich Hochschule und 
Bundesland, die Formulierung konkreter, idealerweise quantifizierbarer Ziele, die Beschränkung auf Ziele in Abgrenzung zu diesen Zielen führenden Maßnahmen, die Dualität von Leistung und Gegenleistung sowie die Individualität des Vertrags (Berthold 2011, 68). Varianten zu diesen Merkmalen können die ausdrückliche Fassung strategischer Ziele oder die in einzelnen Bundesländern vorzufindende Vereinbarung über Mittel für konkrete Projekte darstellen.

Rogal weist auf den Ursprung der Zielvereinbarung aus Unternehmens- und Mitarbeitendenführungsmodellen der Betriebswirtschaft hin (Rogal 2008, 87). Die Bedeutung der Zielvereinbarung kann zum einen in ihrer Grundlagenfunktion für weitere Hochschusteuerungsinstrumente (Schmuck 2010, 349), zum anderen in ihrem seitens der Hochschulleitungen als hoch eingeschätzten Einfluss auf die Strategie der Lehr- und Forschungsanstalten (Winde et al. 2016, 36) festgemacht werden. Unbedingt zu unterscheiden ist die in Kooperation geschlossene Zielvereinbarung von der einseitig ausgesprochenen Zielvorgabe.

In die Untersuchung einbezogen wurden diejenigen frei verfügbaren ESPS und Zielvereinbarungen, die für das Jahr 2017 bzw. an dessen Ende gültig waren. Eine Übersicht über die Zielvereinbarungen $\mathrm{zu}$ jenem Zeitpunkt bietet eine Zusammenstellung der Hochschulrektorenkonferenz (HRK 2017). Die Bundesländer Baden-Württemberg und Sachsen haben die für das Jahr 2017 aktuellen Zielvereinbarungen mit den Hochschulen in ihrer Trägerschaft nicht der Allgemeinheit zugänglich veröffentlicht. Somit setzt sich das Quellenmaterial der Zielvereinbarungen wie in Tabelle 1 aufgeführt zusammen:

Tab. 1: Veröffentlichung der Zielvereinbarungen zwischen Bundesländern und Hochschulen.

\begin{tabular}{llllll}
\hline & Universität & FH & Kunst-HS & Andere & Gesamt \\
\hline Hochschulen & 83 & 105 & 47 & 5 & 240 \\
ZV veröffentlicht & 69 & 83 & 27 & 2 & 181 \\
ZV unveröffentlicht & 14 & 22 & 20 & 3 & 59 \\
\hline
\end{tabular}

Für ESPs liegt keine vergleichbare Übersicht vor, so dass für diese Dokumente eine Internet-Recherche durchgeführt wurde, die eine sich nach Hochschulart und Trägerschaft differenzierende Publikationslage ergab. Hochschulen in konfessioneller und privater Trägerschaft sowie Verwaltungshochschulen publizieren ihre ESPs in nur sehr geringem Ausmaß oder verzichten darauf, ein derartiges Dokument zu erarbeiten. ${ }^{1}$ Bei Hochschulen in staatlicher Trägerschaft außer

176 private Hochschulen, deren ESP bzw. vergleichbares Strategiedokument nicht frei verfügbar im Internet hinterlegt war, wurden mit der Bitte kontaktiert, diesen bzw. dieses für die Arbeit des Forschungsprojekts zur Verfügung zu stellen. Es konnte auf diese Weise kein erheblicher Zuwachs des Quellenmaterials erzielt werden. 
Verwaltungshochschulen gliedert sich die Publikationslage in eine Vielfalt von Kategorien wie frei verfügbar veröffentlichte, nur hochschulintern veröffentlichte, zum Untersuchungszeitpunkt nicht aktuelle oder nicht veröffentlichte ESPs, die in der tabellarischen Aufbereitung in eine unübersichtliche Komplexität der Darstellung münden würden. Die der Analyse zugrundeliegende Stichprobe wurde aus den aktuell gültigen, frei verfügbar veröffentlichten ESPs gebildet, was zu einer Gesamtmenge von 86 ESPs stattlicher Universitäten, Fachhochschulen, Kunsthochschulen und Dualer Hochschulen führt.

Hinsichtlich des Umfangs der Dokumente lassen sich eminente Unterschiede konstatieren. So reicht die Bandbreite der Länge der analysierten ESPS von 15 bis 334 Seiten, was sowohl auf divergierende Vorgaben seitens der Bundesländer als auch auf spezifische Priorisierungen innerhalb der einzelnen Hochschulen zurückzuführen sein dürfte. In Aufbau und Inhalt herrscht bei den ESPs dem Charakter des Dokumententypus entsprechend eine weitaus größere Autonomie der Hochschulen als bei den Zielvereinbarungen, die der Direktive und strukturellen Formatierung der jeweilig zuständigen Landesministerien unterliegen. Die Spezifika der ESPs gründen somit in der Individualität der Institutionen, während sich Diejenigen der Zielvereinbarungen entlang der Vorgaben der Politik ausrichten: so existieren Zielvereinbarungen, die für alle staatlichen Hochschulen eines Bundeslandes von Wirksamkeit sind (Schleswig-Holstein), solche, deren weitestgehend gleichlautender Text nur in ausgewählten Detailangaben an die separaten Gegebenheiten einer Hochschule angepasst werden (z.B. Berlin) sowie solche, die innerhalb einer vorgegebenen Struktur seitens der Hochschulen auszugestalten sind (beispielsweise Bayern oder NordrheinWestfalen). Auf einer inhaltlichen Ebene sind zudem Zielvereinbarungen, die an allgemeine strategische Schwerpunktsetzungen und Entwicklungslinien orientiert sind (z. B. Niedersachen oder Sachsen-Anhalt), von denjenigen zu unterscheiden, die nach einer pauschalen Einleitung auf konkrete Projekte innerhalb der Vereinbarungslaufzeit fokussieren (z. B. Rheinland-Pfalz).

Ungeachtet der Diversität des Quellenmaterials erfolgte dessen Analyse konstant für alle Dokumente unter besonderer Berücksichtigung der studierendenorientierten Perspektive, ausgerichtet auf die vier Untersuchungsdimensionen des Forschungsprojekts Lernwelt Hochschule: Hochschulorganisation, Hochschuldidaktik, Digitale Strukturen und Physischer Lehr- und Lernraum. Dabei wurde eine Inhaltsanalyse vorgenommen, um Strukturen des Materials abzubilden, indem den gesetzten Dimensionen entsprechende Textabschnitte systematisch segregiert, sofern notwendig, paraphrasiert, auf typische Ausprägungen untersucht sowie abschließend je Dimension zusammengefasst und auf Häufigkeiten überprüft wurden (zur Methodik: Aschinger et al. 2020, 39-41; Mayring 2009, 469-473). Konkret wurde nach einer ersten Materialsichtung zur Grobka- 
tegorisierung nach den vier Dimensionen innerhalb der so generierten vier Cluster eine iterative Feinkategorisierung in einzelne inhaltliche Kategorien vollzogen. Diese Subkategorien wurden dann wieder zu Rubriken zusammengefasst, die inhaltlich die jeweils drei anderen Untersuchungsdimensionen abbilden. Die Subkategorien der Dimension Hochschuldidaktik wurden beispielsweise unter den Rubriken Digitale Strukturen, Hochschulorganisation und Physischer Lehr- und Lernraum zusammengeführt. Hochschulorganisation wurde dabei im gesamten Zusammenhang der Dokumentenanalyse nicht als allgemeine Hochschulorganisation, sondern im Sinne der Organisation der Hochschule als Lernwelt sowie der Organisation einer studierenden- bzw. lernendenorientierten Hochschule definiert. Um potentielle Spezifika im Zusammenhang mit Hochschulart und Bundesland in Trägerschaft identifizieren zu können, wurde neben und im Abgleich mit der inhaltlichen, an den Untersuchungsdimensionen orientierten Kategorisierung auch eine solche für die beiden genannten Cluster durchgeführt. Des Weiteren wurden Quantitäten der Textabschnitte, also deren Ausformung als beispielsweise Stichworte, Sätze, Absätze oder Kapiteln erfasst.

Aus der Grobkategorisierung ergab sich auf diese Weise das in Tabelle 2 und $3 \mathrm{zu}$ findende erste Bild:

Tab. 2: Textabschnitte zu den vier Untersuchungsdimensionen in der Grobkategorisierung in Zielvereinbarungen.

\begin{tabular}{llllll}
\hline & Universität & FH & Kunst-HS & Andere & Gesamt \\
\hline Didaktik & 35 & 45 & 9 & 2 & 91 \\
Digitalisierung & 51 & 51 & 18 & 1 & 121 \\
Organisation & 1 & - & 3 & - & 4 \\
Physischer Raum & 41 & 51 & 20 & 1 & 113 \\
\hline
\end{tabular}

Tab. 3: Textabschnitte zu den vier Untersuchungsdimensionen in der Grobkategorisierung in ESPs.

\begin{tabular}{llllll}
\hline & Universität & FH & Kunst-HS & Andere & Gesamt \\
\hline Didaktik & 45 & 71 & 5 & 8 & 129 \\
Digitalisierung & 62 & 66 & 12 & 10 & 150 \\
Organisation & 7 & 2 & - & 1 & 10 \\
Physischer Raum & 60 & 58 & 11 & 2 & 131 \\
\hline
\end{tabular}

Setzt man die Anzahl der Dokumente mit derjenigen der identifizierten Textabschnitte in ein Verhältnis zu einander, fällt auf, dass in den ESPs eine weitaus höhere Anzahl an Textabschnitten vorhanden ist als in den mit mehr als doppelt so vielen Dokumenten in der Untersuchungsgruppe vertretenen Zielvereinbarungen. Dies dürfte in dem potentiell höheren Konkretisierungs-, Spezifikati- 
ons- und Individualisierungsgrad der ESPs im Vergleich zu den Zielvereinbarungen gründen. Da es sich im Fall der Zielvereinbarungen zudem bei 86 der 327 Textabschnitte um solche handelt, die in einer gleichlautenden Formulierung für alle Hochschulen der betreffenden Bundesländer verwendet werden ${ }^{2}$, forciert sich die Differenz um ein weiteres, bezieht man ausschließlich originäre Textabschnitte in den Vergleich ein. Als ebenso evident lässt sich konstatieren, dass sich Textabschnitte zur Untersuchungsdimension Hochschulorganisation in Relation zu den übrigen drei Dimensionen selten nachweisen lassen. Hochschulorganisation wird in den analysierten Dokumenten in einer allgemeinstrukturellen Bedeutung mit Bezug auf etwa Kooperation oder Reorganisation von Gremien oder Abteilungen durchaus thematisiert, in der der vorliegenden Dokumentenanalyse zugrunde gelegten Definition (s.o.) stellt sie hingegen in den behandelten Texten eine Ausnahme dar. Die Reihenfolge der Häufigkeit von Textabschnitten $\mathrm{zu}$ den übrigen drei Untersuchungsdimensionen deckt sich bei ESPs und Zielvereinbarungen: die meisten Textabschnitte können zur Dimension digitale Strukturen (150 in ESPs, 121 in Zielvereinbarungen) identifiziert werden, es folgen die Dimensionen Physischer Lehr- und Lernraum (131 in ESPs, 113 in Zielvereinbarungen) sowie Hochschuldidaktik (129 in ESPs, 91 in Zielvereinbarungen).

Betrachtet man nun die konkrete thematische Ausformung innerhalb der Dimensionen, ergeben sich in der unweigerlich zu Verallgemeinerung und Verlust an Trennschärfe führenden Zusammenfassung für die drei Dimensionen mit einer großen Anzahl von Textabschnitten Befunde wie sie sich in Tabelle 4 finden.

Tab. 4: Textabschnitte zur Untersuchungsdimension Hochschuldidaktik in ESPs und Zielvereinbarungen der Stichprobe.

\begin{tabular}{llll}
\hline & Zielvereinbarungen & ESPs & Gesamt \\
\hline Didaktik allgemein & 5 & 10 & 15 \\
didaktische Qualität & 2 & 4 & 6 \\
didaktische Weiterbildung & 12 & 32 & 44 \\
didaktisches Hochschulkonzept & 3 & 6 & 9 \\
E-Learning, E-Lehre & 6 & 38 & 44 \\
innovative Lehrformen & 11 & 43 & 54 \\
Medieneinsatz & 3 & 2 & 5 \\
Studierendenzentrierung & - & 7 & 7 \\
\hline
\end{tabular}

2 Es handelt sich um die Bundesländer Berlin, Brandenburg, Sachsen-Anhalt und SchleswigHolstein. 
Bei einer Grundgesamtheit von 181 untersuchten Zielvereinbarungen und 86 untersuchten ESPs lassen sich 54 Textabschnitte zu innovativen Lehrformen nachweisen, in denen die Entwicklung, Erprobung oder verstärkte Anwendung neuartiger Lehrformate angekündigt wird. Die Erweiterung des Verständnisses zu Lehr- und Lernformaten bleibt dabei Ausnahme. Gleiches gilt für die Konkretisierung oder inhaltliche Füllung dieser Ankündigungen, es wird lediglich in einem knappen Viertel der Textabschnitte ein Kontext zu E-Learning bzw. E-Lehre gezogen. E-Learning und E-Lehre werden in eigenständiger Form in 44 Textabschnitten im Zusammenhang mit didaktischen Topoi angeführt. Dabei wird entweder eine allgemeine Verstärkung der Aktivität auf diesem Gebiet oder eine Intensivierung der Einbindung dieser Instrumente in didaktische Konzepte in Aussicht gestellt oder auf bereits existente E-Angebote als Nachweis der Innovationskraft der jeweiligen Institution verwiesen. Ebenfalls 44 Textabschnitte können zur didaktischen Fort- und Weiterbildung des Lehrpersonals identifiziert werden, worunter inhaltlich höchst unterschiedliche Resultate von konkreten institutionellen Vorgaben oder Maßnahmen über die Formulierung der Verpflichtung oder Freiwilligkeit der Weiterbildung bis hin zur bloßen stichpunktartigen Erwähnung zu konstatieren sind. In lediglich sieben ESPs erfolgt eine Kontextualisierung von Didaktik und Studierendenorientierung, wobei in allen sieben Fällen eine Ausrichtung didaktischer Konzepte an einer Studierendenorientierung avisiert wird, ohne darzulegen, was dies faktisch bedeutet oder auf welchem Wege dies vollzogen werden soll.

Tab. 5: Textabschnitte zur Untersuchungsdimension Digitale Strukturen in ESPs und Zielvereinbarungen der Stichprobe.

\begin{tabular}{llll}
\hline & Zielvereinbarungen & ESPs & Gesamt \\
\hline Campus Management System & 10 & 10 & 20 \\
Digitale Lehre & 31 & 25 & 56 \\
Digitalisierung allgemein & 11 & 17 & 28 \\
Digitalisierungsstrategie & - & 6 & 6 \\
E-/Blended-Learning & 50 & 40 & 90 \\
Hochschulverwaltung & 2 & 9 & 11 \\
Homepage/Social Media & 1 & 5 & 6 \\
IT-Infrastruktur & 10 & 29 & 39 \\
Medieneinsatz & 8 & 8 & 16 \\
Open Access & 3 & 3 & 6 \\
\hline
\end{tabular}

Innerhalb der Textabschnitte zur Untersuchungsdimension Digitale Strukturen bilden diejenigen zu E- bzw. Blended-Learning mit der Anzahl von 90 das umfangreichste Cluster. Inhaltlich wird zumeist eine Erweiterung oder ein Ausbau 
der diesbezüglichen Angebote und Aktivitäten thematisiert. Dies trifft auch für das mit 56 Textstellen zweitgrößte Cluster Digitale Lehre zu, sodass mehr als die Hälfte aller Resultate der Dimension Digitale Strukturen auf didaktische Aspekte ausgerichtet sind. 39 Textabschnitte beziehen sich auf die IT-Infrastruktur und bilden damit das einzige Cluster, das in einem Kontext mit den Voraussetzungen des physischen Raums für die Digitalisierung steht. Unter den der Organisation zuzuordnenden Clustern stellt Campus Management System mit 20 Textabschnitten das Umfänglichste dar. Hier wird meist die Einführung eines solchen Systems angekündigt. Verweise auf eine hochschulweite Digitalisierungsstrategie lassen sich in lediglich sechs Textabschnitten finden. Auch wenn vereinzelt auf entsprechende Potentiale der Digitalisierung wie Steigerung von Flexibilität oder Mobilität Bezug genommen wird, konstruiert keines der analysierten Dokumente einen Zusammenhang zwischen Digitalisierung und Studierendenorientierung.

Tab. 6: Textabschnitte zur Untersuchungsdimension Physischer Lehr- und Lernraum in ESPs und Zielvereinbarungen der Stichprobe.

\begin{tabular}{llll}
\hline & Zielvereinbarungen & ESPs & Gesamt \\
\hline bauliche Entwicklung & 19 & 39 & 58 \\
Campus & 10 & 15 & 25 \\
einzelne Gebäude & 8 & 2 & 10 \\
Finanzierung & 15 & 3 & 18 \\
Flächenbedarf & 4 & 14 & 18 \\
Infrastruktur & 4 & 20 & 24 \\
Lernräume/Lernzentren & 4 & 17 & 21 \\
Raumbedarf & 5 & 10 & 15 \\
Standortentwicklung & 1 & 5 & 6 \\
technische Ausstattung & 4 & 1 & 5 \\
\hline
\end{tabular}

Die bauliche Entwicklung der Hochschule und damit assoziierte konkrete Baumaßnahmen werden in 58 Textabschnitten ausgeführt. Mit diesem Wert werden die nächstumfangreichsten Cluster um mehr als das Doppelte übertroffen. Das Feld derjenigen Cluster, die in etwa 20 Textabschnitte in sich vereinigen, ist sehr breit, was den Schluss nahelegt, dass innerhalb der Untersuchungsdimension Physischer Lehr- und Lernraum weniger allgemeine, für einen Großteil der Hochschulen zutreffende Trends vorliegen, sondern vielmehr spezifische Bedingungen und Erfordernisse der einzelnen Institutionen angesprochen werden. Immerhin 21 Textabschnitte thematisieren Lernräume oder Lernzentren, wobei in vier Fällen lediglich der Hochschulbibliothek die Funktion als Lernraum zugewiesen wird, allerdings bleibt auch an dieser Stelle wie insgesamt in der Dimen- 
sion Physischer Lehr- und Lernraum eine Bezugnahme auf Konzepte der Studierendenorientierung aus.

In der Zusammenführung aller Ergebnisse ergibt sich folgendes Bild:

1. Sowohl in den ESPs als auch in den Zielvereinbarungen erfolgt die Darlegung der Inhalte mit Bezug zu den vier Untersuchungsdimensionen in der ganz überwiegenden Zahl der Fälle in Form von einzelnen Absätzen oder stichpunktartiger Aufzählung. In den 86 analysierten ESPs finden sich lediglich 37 eigenständige Kapitel sowie in den 181 analysierten Zielvereinbarungen lediglich 44 eigenständige Kapitel zu einer der Dimensionen. Dabei handelt es sich in beinahe gleichen Teilen um Kapitel zu den Dimensionen Digitale Strukturen und Physischer Lehr- und Lernraum, während nur insgesamt vier eigenständige Kapitel zur Hochschuldidaktik und kein Kapitel zur Hochschulorganisation im an dieser Stelle definierten Sinne nachgewiesen werden können.

2. Im Vergleich von ESPS und Zielvereinbarungen besteht ein höherer Detaillierungsgrad seitens der ESPS, was sich auf die größere Freiheit der Hochschulen bei der Ausformulierung der ESPs im Gegensatz zu den in der Struktur durch die Bundesländer determinierten Zielvereinbarungen zurückführen lässt. Obwohl ESPs in geringerer Anzahl in die Analyse einfließen als Zielvereinbarungen, können in Ersteren mehr Textabschnitte zu den Untersuchungsdimensionen identifiziert werden. Die Häufigkeitsstruktur der Textabschnitte zu den Dimensionen deckt sich bei beiden Dokumentenarten, Digitale Strukturen wird am häufigsten thematisiert, es folgen Physischer Lehr- und Lernraum und Hochschuldidaktik; Hochschulorganisation wird im Vergleich zu den anderen Dimensionen äußerst selten behandelt.

3. Im Abgleich der Bundesländer lässt sich konstatieren, dass in den Zielvereinbarungen von Berlin, Brandenburg und Sachsen-Anhalt besonders viele in allen untersuchten Dokumenten des jeweiligen Landes gleichlautende Formulierungen vorkommen. Eigenständige Kapitel zu den Dimensionen lassen sich beinahe ausschließlich in den Zielvereinbarungen Bayerns, Berlins, Hessens, Schleswig-Holsteins und Thüringens finden, während die Streuung bei den ESPs weitaus breiter ausfällt und lediglich Baden-Württemberg mit besonders vielen Kapiteln hervorsticht. Berliner Zielvereinbarungen widmen sich weitaus häufiger der Dimension Digitale Strukturen als Diejenigen der übrigen Bundesländer, in den ESPs sind Digitale Strukturen in den Dokumenten von BadenWürttemberg, Nordrhein-Westfalen und Schleswig-Holstein überdurchschnittlich vertreten. Hinsichtlich der Dimension Physischer Lehr- und Lernraum trifft dies für die Zielvereinbarungen des Landes Hessen sowie für die ESPS des Landes Rheinland-Pfalz zu. Für die Dimension Hochschuldidaktik besteht die einzige Auffälligkeit in einer deutlich über dem Länderschnitt liegenden Thematisierung in den ESPs Baden-Württembergs. 
4. In der Gegenüberstellung der Hochschularten bewegen sich die Häufigkeiten der Textabschnitte zu den Untersuchungsdimensionen und Diejenigen der Kapitel entlang der Gesamtverteilung. Auf formaler Ebene kann festgestellt werden, dass die ESPs von Kunsthochschulen im Schnitt weniger umfangreich als die ESPs der übrigen Hochschulen in staatlicher Trägerschaft ausfallen. Inhaltlich verlaufen die Trennlinien weniger gemäß der Hochschularten, sondern bei den Zielvereinbarungen zwischen den Bundesländern (s. o.) sowie bei den ESPs zwischen der überwiegenden Anzahl der Hochschulen, die sich in ihren Texten mit gängigen Themen wie Gleichstellung, Senkung der Studienabbruchquote oder Wissenstransfer beschäftigen, und einigen wenigen Institutionen wie der RWTH Aachen, der LMU München oder der TU München, die in ihren ESPs vielmehr darum bemüht sind, ihre Alleinstellungsmerkmale in der nationalen und internationalen Wissenschaftslandschaft herauszuarbeiten sowie die gesellschaftliche Relevanz ihrer Forschung aufzuzeigen. Obgleich sich die Gruppe dieser Hochschulen aus im Rahmen der Exzellenzstrategie geförderten Universitäten zusammensetzt, gilt nicht der Umkehrschluss, dass alle sogenannte Exzellenzuniversitäten auf diese Weise abstrahierende ESPs vorlegen.

5. Eine Kontextualisierung von Untersuchungsdimensionen miteinander findet ausschließlich bei den Themen E-Learning und E-Lehre statt. In 34 Textabschnitten in Zielvereinbarungen sowie 41 Textabschnitten in ESPs werden diese Themen in Verschränkung von Aspekten der Hochschuldidaktik mit Aspekten der Digitalen Strukturen und häufig unter Bezugnahme auf sogenannte innovative Lehrformen betrachtet. Zudem stellt der Themenbereich E-Learning, digitale Lehre, Medieneinsatz und innovative Lehrformen sowohl in der Dimension Hochschuldidaktik als auch in der Dimension Digitale Strukturen der am häufigsten fokussierte Topos dar.

6. ESPs und Zielvereinbarungen eint, dass die in ihnen enthaltenen Ausführungen zu den Untersuchungsdimensionen zumeist im Status des Unkonkreten und Abstrakten verbleiben, was heißt, dass Ankündigungen, Vorhaben, Ziele und die Instrumente zur Umsetzung jener in der Regel schlagwortartig benannt, aber nur selten inhaltlich spezifiziert oder ausgefüllt werden. Eine diesbezügliche Ausnahme bilden lediglich mit der Dimension Physischer Lehr- und Lernraum assoziierte Darstellungen konkreter Bauvorhaben.

7. Ein Zusammenhang zwischen Konzepten der Studierendenorientierung und einer der Untersuchungsdimensionen wird ausschließlich in sieben der Dimension Hochschuldidaktik zugeordneten Textabschnitten vorgenommen, wobei dies ausschließlich in ESPs und in keinem der Fälle in einem über einen Absatz hinausgehenden Umfang erfolgt. Überwiegend handelt sich um die schlichte Nennung des Schlagworts Studierendenzentrierung als unbestimmt bleibender didaktischer Topos der jeweiligen Hochschule. Eine kurze inhaltli- 
che Annäherung wird lediglich im ESP der Universität Siegen vollzogen, in dem es heißt:

Ein weiteres Schlüsselkriterium guter Lehre an der Universität Siegen ist die Studierendenzentrierung, d. h. die Lehre vom Lernen neu zu denken und zu gestalten und verstärkt in einen Interaktionsprozess mit den Studierenden zu treten (,Shift from Teaching to Learning“). (Universität Siegen 2017, 20)

\section{Literatur}

Aschinger, F.; Becker, A.; Gageur, N.; Weichert, H. (2020): Forschungsfeld Lernwelt Hochschule. Methodische Zugänge zur Analyse einer differenzierten Struktur. In: A. Becker; R. Stang (Hrsg.): Lernwelt Hochschule. Dimensionen eines Bildungsbereichs im Umbruch. Berlin; Boston: De Gruyter Saur, 20-46.

Becker, A.; Stang, R. (Hrsg.) (2020): Lernwelt Hochschule. Dimensionen eines Bildungsbereichs im Umbruch. Berlin; Boston: De Gruyter Saur.

Berthold, C. (2011): „Als ob es einen Sinn machen würde... “. Strategisches Management an Hochschulen. Gütersloh: Centrum für Hochschulentwicklung.

Deutscher Bundestag (2019): Grundgesetz für die Bundesrepublik Deutschland. Berlin: Deutscher Bundestag.

Dräger, J.; Friedrich, J.-D.; Mordhorst, L.; Müller, U.; Röwert, R. (2017): Hochschulen brauchen Strategien für das digitale Zeitalter. In: Rat für Forschung und Technologieentwicklung (Hrsg.): Zukunft und Aufgaben der Hochschulen. Digitalisierung - Internationalisierung Differenzierung. Wien: LIT, 263-278.

Geiger, R. (2011): Warum brauchen Hochschulen Strategien? Wissenschaftsmanagement 6, 44-48.

HRK (2017): Zielvereinbarungen bzw. Hochschulverträge im Länder- und Hochschulvergleich. Bonn: Hochschulrektorenkonferenz.

Mayring, P. (2009): Qualitative Inhaltsanalyse. In: U. Flick; E. von Kardoff; I. Steinkes (Hrsg.): Qualitative Forschung. Ein Handbuch. Reinbek bei Hamburg: Rowohlt, 468-475.

Meyer-Guckel, V.; Klier, J.; Kirchherr, J.; Winde, M. (2019): Future Skills. Strategische Potenziale für Hochschulen. Essen: Stifterverband für die deutsche Wissenschaft.

Palandt, K. (2015): Zielvereinbarungen zwischen Hochschulen und Ministerium auf der Basis einer beiderseitigen Hochschulentwicklungsplanung. Kann das funktionieren? Das Hochschulwesen 2, 42-46.

Rogal, U. (2008): Hochschulautonomie und Zielvereinbarungen. Hamburg: Dr. Kovac.

Scherm, E.; de Schrevel, M.; Müller, U. M. (2014): Strategisches Universitätsmanagement. Ergebnisse einer Befragung. In: E. Scherm (Hrsg.): Management unternehmerischer Universitäten. Realität, Vision oder Utopie? Augsburg: Rainer Hampp, 98-118.

Schmid, U.; Baeßler, B. (2016): Strategieentwicklung für Hochschulen im digitalen Zeitalter. Berlin, Hochschulforum Digitalisierung.

Schmuck, S. (2010): Zielvereinbarungen im Hochschulbereich. Berlin: Berliner WissenschaftsVerlag.

Tenorth, H.-E.; Tippelt, R (Hrsg.) (2007): BELTZ Lexikon Pädagogik. Weinheim; Basel: Beltz. 
Universität Siegen (Hrsg.) (2017): Hochschulentwicklungsplan der Universität Siegen. https:// www.uni-siegen.de/start/die_universitaet/ueber_uns/hochschulentwicklung/hochschulentwicklungsplan_web_03_2017.pdf.

Winde, M.; Mönikes, R.; Zinke, G. (2016): Hochschulstrategie und Governance. Essen: Stifterverband für die deutsche Wissenschaft. 